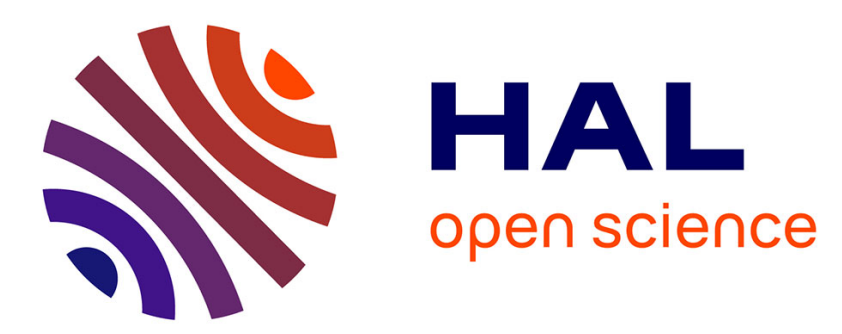

\title{
Robotic Cutting of Soft Materials using Force Control \& Image Moments
}

Philip Long, Wisama Khalil, Philippe Martinet

\section{To cite this version:}

Philip Long, Wisama Khalil, Philippe Martinet. Robotic Cutting of Soft Materials using Force Control \& Image Moments. 2014 13th International Conference on Control Automation Robotics \& Vision (ICARCV), Dec 2014, Singapore, France. pp.474-479, 10.1109/ICARCV.2014.7064351 . hal02461938

\section{HAL Id: hal-02461938 \\ https://hal.inria.fr/hal-02461938}

Submitted on 31 Jan 2020

HAL is a multi-disciplinary open access archive for the deposit and dissemination of scientific research documents, whether they are published or not. The documents may come from teaching and research institutions in France or abroad, or from public or private research centers.
L'archive ouverte pluridisciplinaire HAL, est destinée au dépôt et à la diffusion de documents scientifiques de niveau recherche, publiés ou non, émanant des établissements d'enseignement et de recherche français ou étrangers, des laboratoires publics ou privés. 


\title{
Robotic Cutting of Soft Materials using Force Control \& Image Moments
}

\author{
Philip Long, Wisama Khalil and Philippe Martinet \\ Institut de Recherche en Communications et Cybernétique de Nantes \\ UMR CNRS n 6597 \\ 1 rue de la Noë \\ École Centrale de Nantes, \\ 44321, Nantes, France. \\ Email: Firstname.Lastname@irccyn.ec-nantes.fr \\ Telephone: + 33240376927
}

\begin{abstract}
In this paper, a force/vision control strategy is proposed for the robotic cutting of soft materials. The separation is performed by repeating a series of cuts, called passages, along a deformable three dimensional curve. An image based visual servoing system is proposed to control all six degrees of freedom of the cutting tool. This allows the system to change the cutting depth and angle in response to changes in the profile of the surface. The force is used to ensure the cut is performed cleanly without globally deforming the soft material. The proposed controller is validated experimentally by cutting soft foam material.
\end{abstract}

\section{INTRODUCTION}

Robotic interaction with soft, deformable objects is a challenging and increasingly important topic with applications in several domains [1]. The variability of the object's behavior leads to two classes of solutions. Firstly, the use of advanced object models that can predict the deformation in response to external forces [2]. Secondly, the use of exteroceptive sensors to modify the robot's behavior in order to compensate for the object deformation [3], [4].

The food industry, in particular the meat processing sector, is an area that would benefit from deformable object manipulation solutions. Indeed, existing robotic solutions have resulted in improved hygiene and precision in the manufacturing environment [5]. The ARMS ${ }^{1}$ project, A multi arms Robotic system for Muscle Separation, aims to contribute to the robotization of the meat industry in particular the separation of beef shoulder muscles. A multi-arm system is proposed in order to deal with key challenges such as the flexibility of the target object and its variability [4].

In this study, a force vision controller is proposed for the cutting task. A camera, fixed on the cutting tool, is used to capture an image of the cutting zone. The objective is to change the robot configuration so that the image converges to a desired cutting zone image. This is known as IBVS (Image Based Visual Servoing) in contrast to the more conventional PBVS (Position Based Visual Servoing) where the image is used to reconstruct the 3D-pose of the target object [6]. IBVS is known to have several advantages over PBVS. For

\footnotetext{
${ }^{1}$ arms.irccyn.ec-nantes.fr
}

example IBVS is more robust with respect to calibration errors whereas in PBVS, due to the reconstruction of the 3D position, these errors directly affect the task convergence. Moreover, by controlling the system in the image space, the IBVS system can ensure the image always remains in the field of view [7], [8]. On the other hand, in order to follow an image trajectory the end effector may have to execute unfeasible Cartesian velocities. Furthermore a poorly chosen image feature may lead to a singularity of the interaction matrix.

Image moments provide a robust description of a diverse set of image objects and have been widely used in computer vision tasks [9]. Recently image moments have been applied to visual servoing schemes. By careful combination of image moments, an interaction matrix of maximal decoupled structure and low condition number can be built for a variety of complex images [10], [11], [12].

Generally, force/vision research has been focused on contour following tasks where a force is applied normal to the surface. If the surface is unknown, its pose with respect to the tool must be obtained. By studying the force sensors measurements and approximating the frictional effects of the motion, the normal to the surface can be constructed [13], [14], [15]. The disadvantage of this approach is that the orientation of the tool relies on noisy force measurements and requires a filtering operation to obtain an accurate solution [16]. Moreover in cutting applications this approach is no longer valid, since in order to separate the object the tool must necessarily pass through the contour. In [17], the normal to the surface is obtained using vision data by using an external camera observing the scene. However, these so called eye-tohand systems are limited due to problems regarding spatial resolution and limited field of view [18].

In this paper, we propose a force/vision controller capable of cutting objects while following a 3D deformable trajectory. In contrast to previous works, the orientation of the tool is controlled using an eye-in-hand camera. Therefore, the use of noisy force measurements in the precise positioning task is avoided. In addition to this, by using a local camera instead of an external system, a clear unimpeded view of the desired 
trajectory is provided. For the separation of deformable objects, a local view is essential due its inherent flexibility.

The paper is organized in the following way. In Section II, the robotic cell and notation are described. In Section III, IBVS strategy is outlined. In Section IV the global control scheme is illustrated. In Section V the results are given. Finally, in Section VI the conclusions are drawn and future work is discussed.

\section{Robotic CELL}

A 7-DOF Kuka LWR robot is used for this experiment, as shown in Fig.1. The robot is equipped with an ATI gamma 6-axis force sensor a marlin 1394 camera and a razor blade.

The Modified Denavit-Hartenberg (MDH) notation [19] is used to describe the geometry of the system. The following geometric and kinematic models are obtained:

$$
{ }^{0} \mathbf{T}_{t}=\left[\begin{array}{cc}
{ }^{0} \mathbf{R}_{t} & { }^{0} \mathbf{p}_{t} \\
0 & 1
\end{array}\right], \quad \quad \mathbf{V}_{t}={ }^{0} \mathbf{J}_{t} \dot{\mathbf{q}}
$$

The $4 \times 4$ transformation matrix ${ }^{i} \mathbf{T}_{j}$ represents the location of frame $\mathcal{R}_{j}$ with respect to frame $\mathcal{R}_{i}$ consisting of the $3 \times 3$ rotation matrix ${ }^{i} \mathbf{R}_{j}$ and the $3 \times 1$ position vector ${ }^{i} \mathbf{p}_{j} .{ }^{i} \mathbf{V}_{j}$ denotes the kinematic screw of frame $\mathcal{R}_{j}$ with respect to the fixed world frame and represented in frame $\mathcal{R}_{i} .{ }^{i} \mathbf{J}_{j}$ is the kinematic Jacobian matrix of the robot evaluated at frame $\mathcal{R}_{i}$ and represented in frame $\mathcal{R}_{i} . \mathbf{q}$ and $\dot{\mathbf{q}}$ are the vectors of joint positions and velocities respectively.

Three coordinate frames are defined for this work. $\mathcal{R}_{0}$ is the fixed world frame. $\mathcal{R}_{t}$ is frame fixed to the razor blade. $\mathcal{R}_{c}$ is fixed to the camera where the $z$ axis is aligned with the camera's optical axis. The transformation matrix ${ }^{t} \mathbf{T}_{c}$, the camera with respect to the tool frame, is obtained through offline calibration methods. Finally, it should be noted that the camera frame is parallel to the frame of the cutting tool.

\section{Image Based Visual Servoing}

The cutting trajectory is composed of a series of dense objects located along an unknown curve that varies in three dimensions as shown in Fig.1. The visual controller is used to position all six degrees of freedom of the end effector by ensuring that the extracted image feature $\mathbf{s}$ converges to a desired image feature $\mathbf{s}^{*}$. The desired image, learned off-line using the teach by showing method, specifies the reference cutting frame, including the desired depth and the cutting angle. The camera velocity is found from:

$$
{ }^{c} \mathbf{V}_{c}=\mathbf{\Lambda} \mathbf{L}_{s}^{+}\left(\mathbf{s}^{*}-\mathbf{s}\right)
$$

where $\mathbf{L}_{s}$ is the interaction matrix. ${ }^{c} \mathbf{V}_{c}$ is the camera velocity composed of a $3 \times 1$ vector of linear velocity ${ }^{c} \mathbf{v}_{c}=$ $\left[\begin{array}{lll}v_{x} & v_{y} & v_{z}\end{array}\right]^{T}$ and a $3 \times 1$ vector of angular velocity ${ }^{c} \boldsymbol{\omega}_{c}=\left[\begin{array}{lll}\omega_{x} & \omega_{y} & \omega_{z}\end{array}\right]^{T} . \boldsymbol{\Lambda}$ is an adaptive gain matrix. In the following the image primitive which are used for the IBVS control scheme are described.

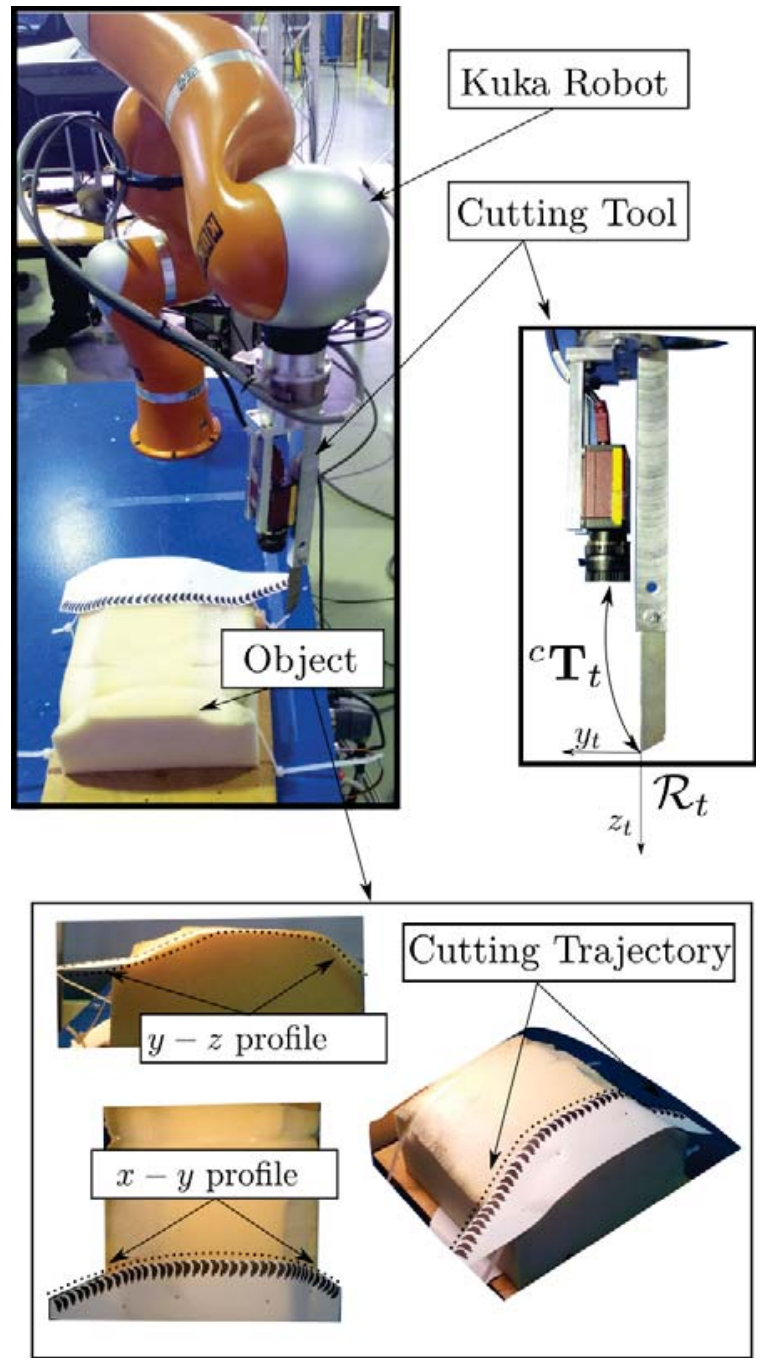

Fig. 1. Experimental Platform where the surface curvature is described in the fixed world frame

\section{A. Image Control Algorithm}

The flow diagram in Fig.2 describes the steps taken to ensure the knife follows a trajectory rather than simply converging to a desired pose. The control scheme selects an object and binarizes the image such that the pixels within the objects boundaries are given an intensity value $I=1$. The remaining part of the image, including other objects in the field of view, are given a value of $I=0$. Once the object has converged to the desired position, the next object on the trajectory is selected. The image moments are extracted for the segmented object as described in the following section.

\section{B. Image Moments}

Image moments can describe a diverse shape of objects after segmentation or binarization. For a binary image, with pixel coordinates $(x, y)$, where the object of interest has intensity 


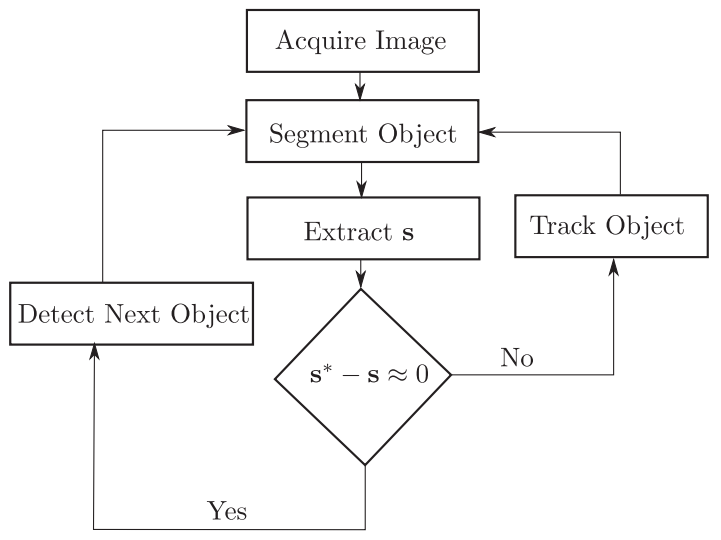

Fig. 2. Image Segmentation Process

$I(x, y)=1$, the raw moments are defined by:

$$
m_{i j}=\sum_{k=1}^{n} x_{k}^{i} y_{k}^{j}
$$

The central moments are defined as :

$$
\mu_{i j}=\sum_{k=1}^{n}\left(x_{k}-x_{g}\right)^{i}\left(y_{k}-y_{g}\right)^{j}
$$

where $x_{g}$ and $y_{g}$ are defined as the center of gravity of the object:

$$
x_{g}=\frac{m_{10}}{m_{00}}, \quad y_{g}=\frac{m_{01}}{m_{00}}
$$

Recently, image moments have been successfully used in visual servoing applications [10]. The image moments are selected to ensure that both the linear and angular velocity of the camera can be controlled. Furthermore they are chosen such that the condition number of the resulting interaction matrix is low. In order to control the cutting tool the following image moments are selected:

$$
\mathbf{s}=\left[\begin{array}{llllll}
x_{n} & y_{n} & a_{n} & \tau_{x} & \tau_{y} & \alpha
\end{array}\right]
$$

The first three components are known as the normalized coordinates of center of gravity and the normalized area [11]. These components are used to control the linear velocity of the camera, they are defined as:

$$
x_{n}=a_{n} x_{g} \quad y_{n}=a_{n} y_{g} \quad a_{n}=z^{*} \sqrt{\frac{a^{*}}{a}}
$$

where $a^{*}$ and $z^{*}$ represent the desired area and the distance between the object and the camera in the desired configuration respectively.

The angular velocity, $\omega_{z}$, about the optical axis of the camera is controlled by the component $\alpha$. This feature is calculated using the central moments from (4) as:

$$
\alpha=\frac{1}{2}\left(2 \frac{\mu_{11}}{\mu_{20}-\mu_{02}}\right)
$$

Finally, in order to control the velocities $\omega_{x}$ and $\omega_{y}$, a careful selection of the image moments $\tau_{x}, \tau_{y}$ must be carried out with respect to the task, the object and the desired object.

Referring to Fig.1, it can be seen that in order to keep the object parallel to the image plane the camera must undergo large rotations around its $x$ axis. Therefore, using the off-line selection method proposed in [20] the following pair of image moments are selected:

$$
\tau_{x}=\frac{I_{3}}{I_{4}} \quad \tau_{y}=\frac{I_{14}}{I_{15}}
$$

$I_{3}, I_{4}, I_{14}$ and $I_{15}$ are four invariant image moments given in [20] calculated using (3) and (4) and based on those originally obtained in [9]:

$$
\begin{aligned}
I_{3} & =\left(\mu_{30}-3 \mu_{12}\right)^{2}+\left(3 \mu_{21}-\mu_{03}\right)^{2} \\
I_{4} & =\left(\mu_{30}+\mu_{12}\right)^{2}+\left(\mu_{21}+\mu_{03}\right)^{2} \\
I_{14} & =\left(\mu_{50}-2 \mu_{32}-3 \mu_{14}\right)^{2}+\left(\mu_{05}-2 \mu_{23}-3 \mu_{41}\right)^{2} \\
I_{15} & =\left(\mu_{50}-10 \mu_{32}+5 \mu_{14}\right)^{2}+\left(\mu_{05}-10 \mu_{23}+5 \mu_{41}\right)^{2}
\end{aligned}
$$

$\tau_{x}$ and $\tau_{y}$ are invariant to translational motions and to rotations around the optical axis [10].

\section{Interaction Matrix}

As shown in (2) an interaction matrix is required to relate the error in the image features to the camera velocity. The chosen interaction matrix is computed from the desired image, $\mathbf{s}^{*}$ where the desired object is parallel to the image plane. By ensuring the desired image is parallel to the image, the interaction matrix is greatly simplified.

The use of a constant intersection matrix, computed using the desired configuration, is justified since the trajectory consists of a series of objects very close to each other. Thus the current object is unlikely to be outside the localized area of convergence. By making the above simplifications an interaction matrix is obtained with the following form:

$$
\mathbf{L}_{\mathbf{s}=\mathbf{s}^{*}}^{\|}=\left[\begin{array}{cccccc}
-1 & 0 & 0 & x_{n \omega x} & x_{n \omega y} & y_{n}^{*} \\
0 & -1 & 0 & y_{n \omega x} & y_{n \omega y} & -x_{n}^{*} \\
0 & 0 & -1 & a_{n \omega x} & a_{n \omega y} & 0 \\
0 & 0 & 0 & \tau_{x \omega x} & \tau_{x \omega y} & 0 \\
0 & 0 & 0 & \tau_{y \omega x} & \tau_{y \omega y} & 0 \\
0 & 0 & 0 & \alpha_{\omega x} & \alpha_{\omega y} & -1
\end{array}\right]
$$

The analytic expressions of the quantities in (14) can be found in [10], [11], however the decoupled structure of the matrix is more important. A particularly interesting feature is the direct link between the distance to the object i.e. the cutting depth and the area of the image object. In the following section, this relationship is used to implement a force controller.

\section{Global Control Strategy}

\section{A. Robot Controller}

An overview of the global robotic control strategy is given in Fig.3. The control scheme is designed to move the camera such that the next image in the trajectory sequence converges to the desired image, as illustrated in Fig.4. If the tool exits the 


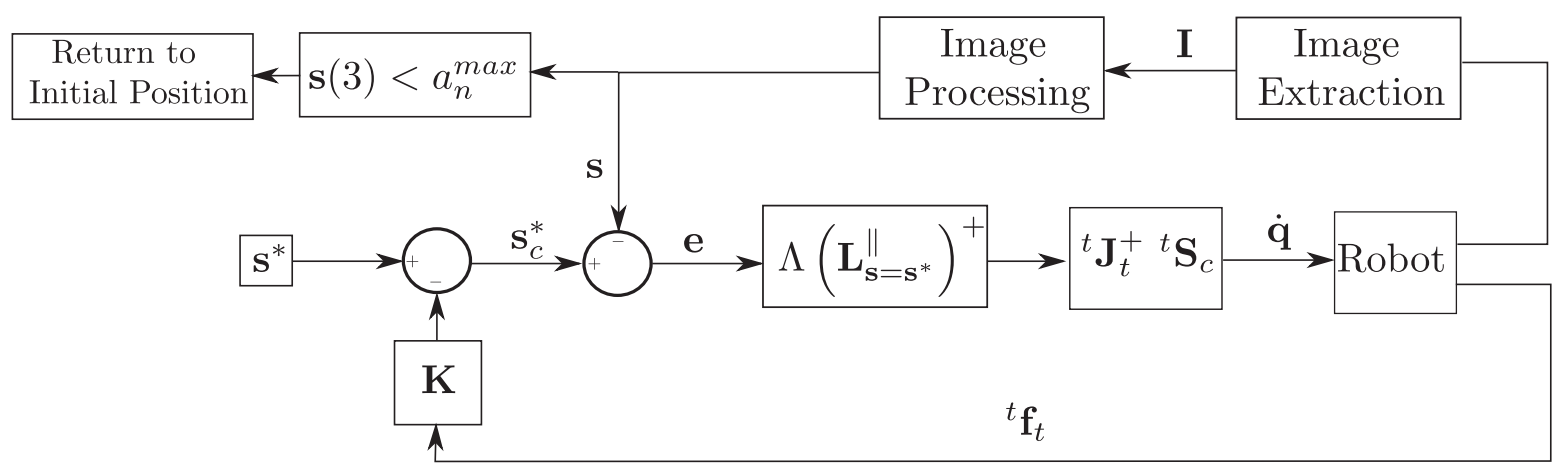

Fig. 3. Global Control scheme
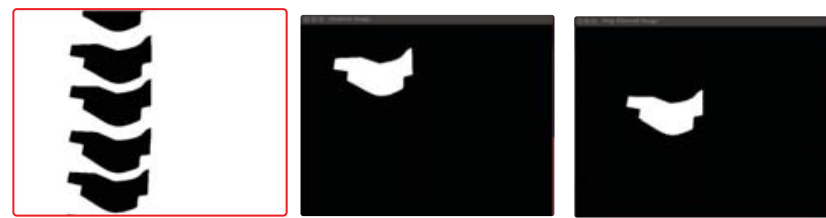

Fig. 4. (a) Extracted scene at time $t$, (b) Desired Image, (c) Current Image

material, the robot returns to the initial position. The robot's movement from the initial position to the moment the tool leaves the media is denoted as a passage. In order to overcome the discrete nature of the trajectory, an adaptive gain matrix $\boldsymbol{\Lambda}$ in (2) is used to increase the rate of convergence as the error decreases.

\section{B. Force Controller}

The force controller is used to ensure that excessive resistive forces are avoided during to the cut [21]. The resistive forces oppose the motion of the tool i.e. they act along the $y$-axis of the tool. We propose to link the resistive force directly to the desired image features as:

$$
\delta a_{n}^{*}=\min \left(0, \quad k_{z}{ }^{t} f_{y}\right)
$$

In this case the min function is used to prevent the knife entering deeper into the material.

\section{EXPERIMENTAL VALIDATION}

\section{A. Experimental Setup}

The experimental setup is shown in Fig.1. A $200 \mathrm{~mm} \times$ $200 \mathrm{~mm} \times 100 \mathrm{~mm}$ block of foam known as Bultex (C) is used. The foam is pre-cut to create an irregular surface. A series of identical objects are adhered to the foam in a curvilinear configuration. The curve varies in the $x, y$ and $z$ directions. The desired image is shown in Fig.4. At the desired configuration, the object is parallel to the image plane and the distance is given as $z^{d}=0.08$. The numerical value of the interaction

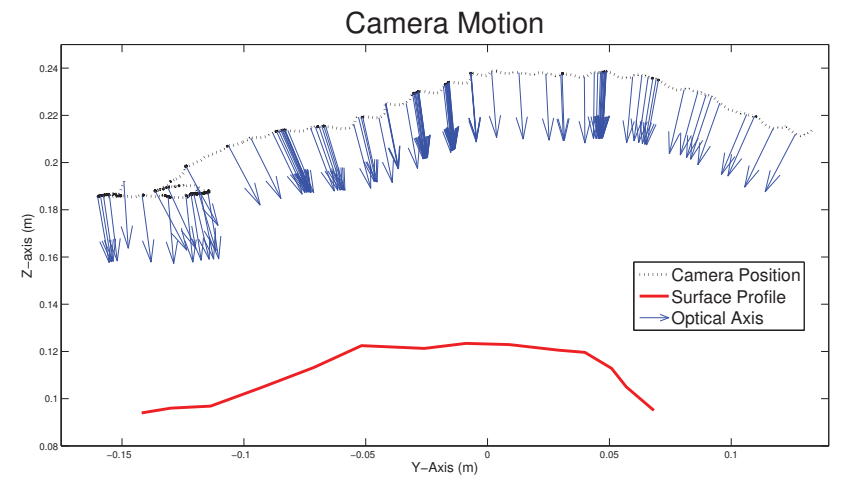

Fig. 5. Camera Trajectory with optical axis in the $y-z$ plane in the fixed world frame

matrix is given as:

$$
\mathbf{L}_{\mathbf{s}=\mathbf{s}^{*}}^{-1}=\left[\begin{array}{cccccc}
-1 & 0 & 0 & 0.016 & 0.071 & 0.007 \\
0 & -1 & 0 & 0.030 & -0.297 & -0.008 \\
0 & 0 & -1 & 0.006 & -0.028 & 0 \\
0 & 0 & 0 & 0.385 & -3.687 & 0 \\
0 & 0 & 0 & -0.194 & -0.867 & 0 \\
0 & 0 & 0 & -0.044 & -0.056 & -1
\end{array}\right]
$$

while the value of the desired image feature is given as:

$\mathbf{s}^{*}=\left[\begin{array}{llllll}-8.04 & -7.0 & 80 & 11067 & 1038.0 & 38.556\end{array}\right] \times 10^{3}$

\section{B. Experimental Results}

Fig. 5 gives the evolution of the camera position in the $y-z$ plane, the surface profile and the orientation of the optical axis. It can be seen that the orientation of camera's optical axis is adjusted throughout the trajectory in order to keep the object parallel to the image plane.

Fig.6 shows the resistive force due to the cutting action. As the force increases the controller induces a change in the desired normalized area as described by (15). This slicing action then results in a drop in the magnitude of the force. This is particularly clear for passage 10 .

Finally, Fig.7 shows the error of the image moments for two consecutive objects. It can be seen that all the image features 

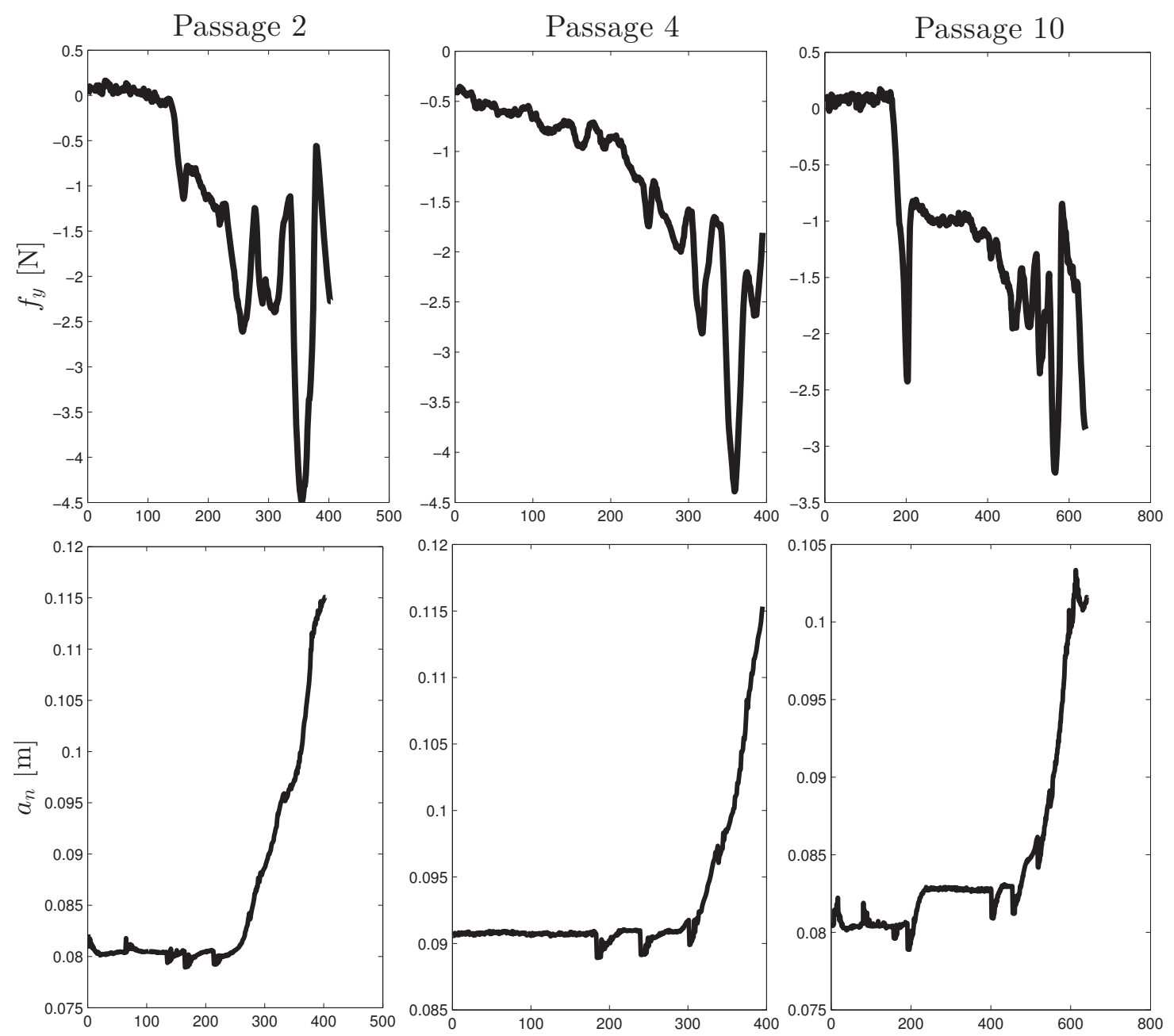

Fig. 6. Deviation due to resistive cutting force

converge to their desired values. The spikes in the graphs at iteration number 2300 and 3400 indicate the detection of the next object in the trajectory and thus an instantaneous change in the value of $\mathbf{s}$ as described in Fig.2. It is also clear from Fig.7 that the convergence of $\tau_{x}$ and $\tau_{y}$ is much slower than the other image features.

\section{CONCLUSION}

In this paper, a force/vision control strategy was proposed for the robotic cutting of soft materials. In contrast to previous works, a local vision system is used to control the angle between the surface and the cutting tool thereby eliminating the effect of noisy force measurements on the orientation task. Furthermore, this system allows the cutting tool to follow a three dimensional surface while maintaining a constant cutting depth.

By controlling the system using IBVS and by adding the force deviation directly to the image space, the sensitivity to calibration errors is reduced. However since discrete objects are used to represent a continuous cutting trajectory, the convergence of the image features may lead to an unsatisfac- tory cutting trajectory. In the cutting task, this could lead to unnecessary forces on the tool. In future work, we will address this issue by firstly increasing camera resolution, allowing the discrete objects to better approximate a continuous trajectory and secondly, taking into account the next object in the control law.

\section{ACKNOWLEDGMENT}

This work was carried out in the framework of the ARMS project, a project funded by the ANR (Agence Nationale de la Recherche), reference ANR-10-SEGI-000. Parts of the equipment used here were funded by the project ROBOTEX, reference ANR-10-EQPX-44-01.

\section{REFERENCES}

[1] M. Saadat and P. Nan, "Industrial applications of automatic manipulation of flexible materials," Industrial Robot: An International Journal, vol. 29, no. 5, pp. 434-442, 2002.

[2] D. Sun and Y. Liu, "Modeling and impedance control of a twomanipulator system handling a flexible beam," in Robotics and Automation, 1997. Proceedings., 1997 IEEE International Conference on, vol. 2, pp. 1787-1792 vol.2, 1997. 

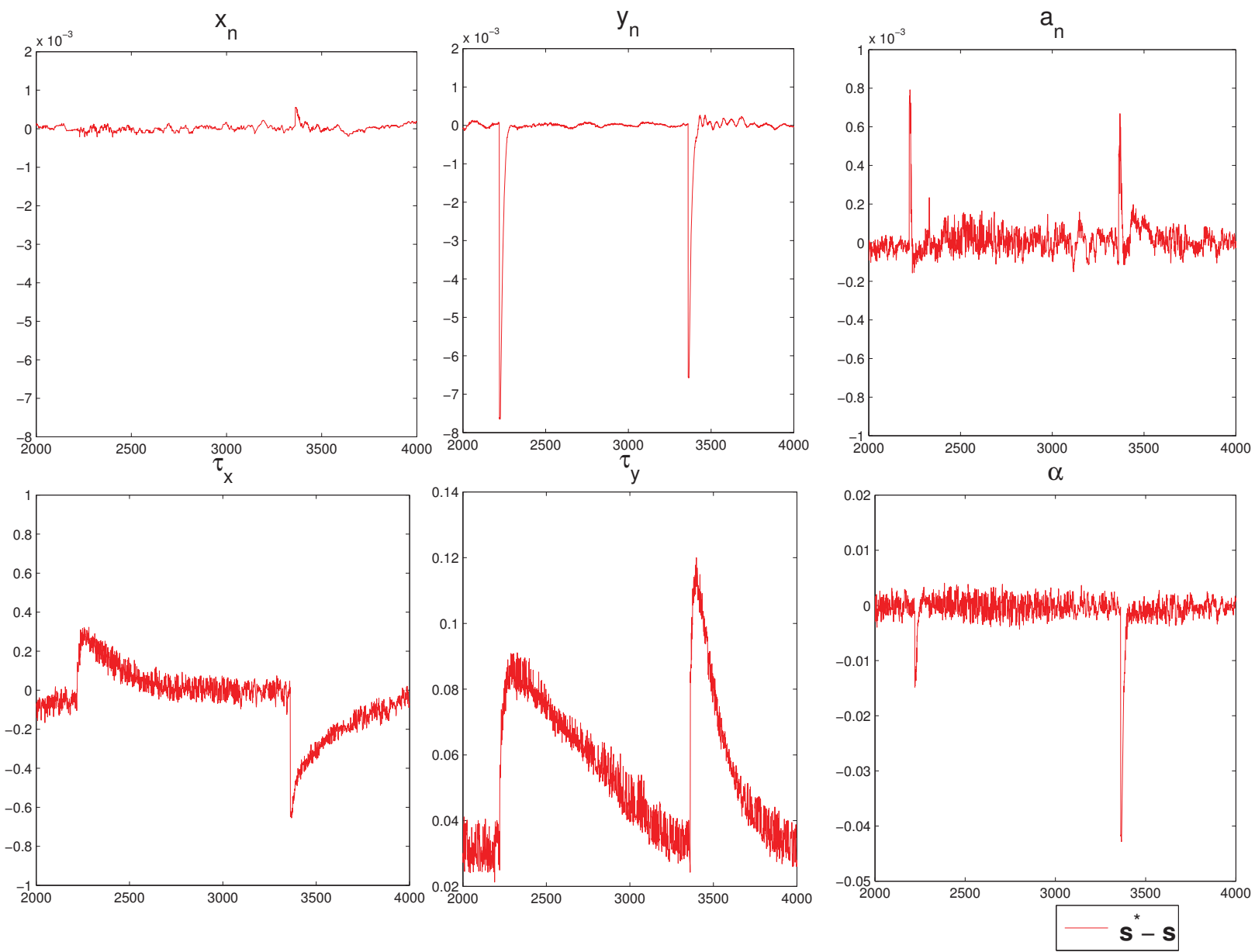

Fig. 7. Error of all image moments during point to point trajectory

[3] D. Navarro-Alarcón, Y.-H. Liu, J. G. Romero, and P. Li, "Modelfree visually servoed deformation control of elastic objects by robot manipulators," IEEE Transactions on Robotics and Automation, vol. 29, pp. 1457-1468, dec 2013.

[4] P. Long, W. Khalil, and P. Martinet, "Modeling and control of a meatcutting robotic cell," in 2013 15th International Conference on Advanced Robotics (ICAR), pp. 61-66, IEEE, 2013.

[5] L. Hinrichsen, "Manufacturing technology in the danish pig slaughter industry," Meat science, vol. 84, no. 2, pp. 271-275, 2010

[6] F. Chaumette and S. Hutchinson, "Visual servo control. II. Advanced approaches [Tutorial]," IEEE Robotics \& Automation Magazine, vol. 14, pp. 109-118, Mar. 2007.

[7] F. Chaumette, "Potential problems of stability and convergence in imagebased and position-based visual servoing," in The confluence of vision and control, pp. 66-78, Springer, 1998.

[8] P. I. Corke and S. A. Hutchinson, "A new partitioned approach to imagebased visual servo control," Robotics and Automation, IEEE Transactions on, vol. 17, no. 4, pp. 507-515, 2001.

[9] M.-K. Hu, "Visual pattern recognition by moment invariants," IRE Transactions on Information Theory, vol. 8, no. 2, pp. 179-187, 1962.

[10] F. Chaumette, "Image moments: a general and useful set of features for visual servoing," IEEE Transactions on Robotics, vol. 20, no. 4, pp. 713$723,2004$.

[11] O. Tahri and F. Chaumette, "Point-based and region-based image moments for visual servoing of planar objects," IEEE Transactions on Robotics, vol. 21, no. 6, pp. 1116-1127, 2005.

[12] C. Copot, A. Burlacu, and C. Lazar, "Visual predictive control architecture based on image moments for manipulator robots," in Proceedings of the 2011 IEEE International Symposium on Industrial Electronics (ISIE), pp. 963-968, IEEE, 2011.
[13] K. Hosoda, K. Igarashi, and M. Asada, "Adaptive hybrid control for visual and force servoing in an unknown environment," Robotics \& Automation Magazine, IEEE, vol. 5, no. 4, pp. 39-43, 1998.

[14] A. Leite, F. Lizarralde, and L. Hsu, "Hybrid vision-force robot control for tasks on unknown smooth surfaces," in Robotics and Automation, 2006. ICRA 2006. Proceedings 2006 IEEE International Conference on, pp. $2244-2249$, may 2006.

[15] A. Pichler and M. Jagersand, "Uncalibrated hybrid force-vision manipulation," in Proceedings of the 2000 International Conference on Intelligent Robots and Systems, (IROS 2000), vol. 3, pp. 1866-1871 vol.3, 2000.

[16] D. Xiao, B. Ghosh, N. Xi, and T. Tarn, "Sensor-based hybrid position/force control of a robot manipulator in an uncalibrated environment," IEEE Transactions on Control Systems Technology, vol. 8, no. 4, pp. 635-645, 2000.

[17] W.-C. Chang, "Hybrid force and vision-based contour following of planar robots," Journal of Intelligent \& Robotic Systems, vol. 47, pp. 215237, 2006. 10.1007/s10846-006-9074-0.

[18] J. A. Piepmeier and H. Lipkin, "Uncalibrated eye-in-hand visual servoing," The International Journal of Robotics Research, vol. 22, no. 10-11, pp. 805-819, 2003.

[19] W. Khalil and J. Kleinfinger, "A new geometric notation for open and closed-loop robots," in Proceedings. 1986 IEEE International Conference on Robotics and Automation, vol. 3, pp. 1174-1179, IEEE, 1986.

[20] O. Tahri, Application des moments à l'asservissement visuel et au calcul de pose. $\mathrm{PhD}$ thesis, Université de Rennes, 2004.

[21] P. Long, W. Khalil, and P. Martinet, "Force/vision control for robotic cutting of soft materials," in 2014 IEEE/RSJ International Conference on Intelligent Robots and Systems (IROS), 2014, 2014. 